\title{
GERMS OF LOCAL AUTOMORPHISMS OF REAL-ANALYTIC CR STRUCTURES AND ANALYTIC DEPENDENCE ON $k$-JETS.
}

\author{
DMITRI ZAITSEV
}

\begin{abstract}
The topic of the paper is the study of germs of local holomorphisms $f$ between $\mathbb{C}^{n}$ and $\mathbb{C}^{n^{\prime}}$ such that $f(M) \subset M^{\prime}$ and $d f\left(T^{c} M\right)=T^{c} M^{\prime}$ for $M \subset \mathbb{C}^{n}$ and $M^{\prime} \subset \mathbb{C}^{n^{\prime}}$ generic real-analytic CR submanifolds of arbitrary codimensions. It is proved that for $M$ minimal and $M^{\prime}$ finitely nondegenerate, such germs depend analytically on their jets. As a corollary, an analytic structure on the set of all germs of this type is obtained.
\end{abstract}

\section{Introduction}

Let $M \subset \mathbb{C}^{n}, M^{\prime} \subset \mathbb{C}^{n^{\prime}}$ be connected locally closed real-analytic submanifolds, $x \in M, x^{\prime} \in M^{\prime}$ be arbitrary points. The complex tangent subspace $T_{x} M \cap i T_{x} M$ will be denoted by $T_{x}^{c} M$. $M$ is a $C R$ manifold, if $\operatorname{dim} T_{x}^{c} M$ is constant. In this case $\operatorname{dim}_{\mathrm{CR}} M:=\operatorname{dim}_{\mathbb{C}} T^{c} M$ is called the $C R$ dimension and $\operatorname{codim}_{\mathrm{CR}} M:=\operatorname{dim}_{\mathbb{R}} T M-\operatorname{dim}_{\mathbb{R}} T^{c} M$ the $C R$ codimension. A CR submanifold $M \subset \mathbb{C}^{n}$ is called generic, if $T M+i T M=\mathbb{C}^{n}$.

Suppose for the moment that $M, M^{\prime} \subset \mathbb{C}^{n}$ are generic real-analytic $\mathrm{CR}$ submanifolds of the same CR dimension and the same CR codimension. Baouendi, Ebenfelt and Rothschild found optimal nondegeneracy conditions on $M$ and $M^{\prime}$ such that a germ at $x$ of a local biholomorphism $f$ (between some neighborhoods in $\mathbb{C}^{n}$ ) with $f(M) \subset M^{\prime}, f(x)=x^{\prime}$, is uniquely determined by its $k$-jet $j_{x}^{k} f$, where $k$ is an integer which depends only on $M$ and $M^{\prime}$ (see [2], Theorem 1, Proposition 2.3). A similar statement for the case of hypersurfaces $M$ and $M^{\prime}$ is implicitly contained in [8]. See also [5] for the case of Levi-nondegenerate higher codimensional CR manifolds. These results should be compared with the following theorem of H. Cartan $([6,11])$ :

Let $D \subset \mathbb{C}^{n}$ be a bounded domain. Then the group of all biholomorphic automorphisms Aut $(D)$ equipped with the compact-open topology is a Lie group. Moreover, if $x \in D$ is arbitrary, an automorphism $f \in \operatorname{Aut}(D)$ is uniquely determined by its 1-jet $j_{x}^{1} f$ and depends analytically on it.

Received February 7, 1997. Revised August 10, 1997.

1991 Mathematics Subject Classification. 32C16, 32D15.

Partially supported by SFB 237 "Unordnung und große Fluktuationen" 
In the above CR setting it is not clear how a map (germ) $f$ depends on its $k$-jet $j_{x}^{k} f$. Is it somehow continuous, analytic etc.?

The special case when both $M$ and $M^{\prime}$ are Levi-nondegenerate hypersurfaces was previously considered by Tanaka [14] and Chern-Moser [7], where the determinacy of automorphisms by their 2-jets was shown. Even in this special context an analytic dependence on the jets is of interest. An algebraic dependence of the automorphisms of bounded domains on their 1-jets is studied in [18], which can be seen as an algebraic version of the above theorem of Cartan.

The present paper has a different point of view. Instead of global automorphisms we consider locally defined germs of holomorphic maps $f$ sending $M$ into $M^{\prime}$ with no assumptions on their domains of definition. If either $M$ or $M^{\prime}$ is locally biholomorphic to $\mathbb{R}^{s} \times \mathbb{C}^{l}$, the space of such germs is infinite-dimensional (see also [2], Theorem 3) and hence it cannot be parametrized by a finite dimensional $k$-jet space.

Therefore both $M$ and $M^{\prime}$ have to satisfy certain nondegeneracy conditions. We start with some stronger conditions formulated in terms of the Levi form. Recall that in the case of arbitrary CR codimension the Levi form is a sesquilinear map (see $\S 2$ ):

$$
L: T_{x}^{1,0} M \times T_{x}^{1,0} M \rightarrow\left(T_{x} M / T_{x}^{c} M\right) \otimes_{\mathbb{R}} \mathbb{C} .
$$

Definition 1.1. We say that the Levi form at $x$ is nondegenerate if for $X_{0} \in$ $T_{x}^{1,0} M$,

$$
\left(L\left(X_{0}, Y\right)=0 \forall Y \in T_{x}^{1,0} M\right) \quad \Longrightarrow \quad X_{0}=0 .
$$

The Levi form is said to be surjective at $x$, if the map (1) is surjective. The Levi form is said to be nondegenerate (resp. surjective) if it is nondegenerate (resp. surjective) for all $x \in M$.

Now we introduce the notion of analytic dependency on $k$-jets. Let $S$ be a subset of the set of germs at $x \in \mathbb{C}^{n}$ of all local holomorphic maps $f$ between $\mathbb{C}^{n}$ and $\mathbb{C}^{n^{\prime}}$. By $J_{x}^{k}\left(\mathbb{C}^{n}, \mathbb{C}^{n^{\prime}}\right)$ denote the $k$-jet space at $x$ of such maps and by $j_{x}^{k} f \in J_{x}^{k}\left(\mathbb{C}^{n}, \mathbb{C}^{n^{\prime}}\right)$ the $k$-jet of $f$ at $x$. We write also $J^{k}\left(\mathbb{C}^{n}, \mathbb{C}^{n^{\prime}}\right)$ for the (trivial) bundle of all $k$-jets at all $x \in \mathbb{C}^{n}$.

Definition 1.2. We say that the germs in $S$ depend analytically on their $k$-jets at $x$ if the following conditions are satisfied:

(1) $f \in S$ is uniquely determined within $S$ by $j_{x}^{k} f$, i.e. for $f_{1}, f_{2} \in S, j_{x}^{k} f_{1}=$ $j_{x}^{k} f_{2} \quad \Longrightarrow \quad f_{1}=f_{2}$;

(2) For every $f_{0} \in S$, there exist neighborhoods $U(x) \subset \mathbb{C}^{n}, U\left(j_{x}^{k} f_{0}\right) \subset$ $J_{x}^{k}\left(\mathbb{C}^{n}, \mathbb{C}^{n^{\prime}}\right)$ such that every $f \in S$ with $j_{x}^{k} f \in U\left(j_{x}^{k} f_{0}\right)$ extends holomorphically to $U(x)$;

(3) In addition there exists a holomorphic map $F: U\left(j_{x}^{k} f_{0}\right) \times U(x) \rightarrow \mathbb{C}^{n^{\prime}}$ such that for all $f \in S$ with $j_{x}^{k} f \in U\left(j_{x}^{k} f_{0}\right)$,

$$
f(z)=F\left(j_{x}^{k} f, z\right), z \in U(x) .
$$


For $x \in \mathbb{C}^{n}$, we equip $S$ with the inductive limit topology, i.e. a sequence $\left(f_{n}\right)$ in $S$ converges to $f_{0} \in S$ if and only if all $f_{n}$ extend to some neighborhood of $x$ and converge there to $f_{0}$ uniformly.

Theorem 1.1. Suppose that the Levi form of $M$ is nondegenerate and surjective. Then there exists an integer $k>0$ such that for all $x \in M$, the germs at $x$ of local biholomorphisms $f$ with $f(M) \subset M$ depend analytically on their $k$-jets. One can take $k=2\left(1+\operatorname{codim}_{\mathrm{CR}} M\right)$.

As an application we obtain a Lie group structure on the set of germs fixing a point $x \in M$ (see the end of the section for the proof).

Corollary 1.1. Let $x \in M$ be arbitrary and the Levi form of $M$ be nondegenerate and surjective at $x$. Then the group of all germs of local biholomorphisms $f$ with $f(M) \subset M, f(x)=x$, is a Lie group.

Notice that the domain of definition of a germ $f$ can vary as $j_{x}^{k} f$ changes. It is not even clear a priori whether a 1-parameter family $f_{t}, t \in \mathbb{R}$, of such automorphisms yields a germ of a vector field. The analytic dependence on the $k$-jets guarantees, in particular, the following extension result for the germs $f$ with $j_{x}^{k} f$ sufficiently close to $j_{x}^{k} f_{0}$ for $f_{0}$ given.

Corollary 1.2. Under the assumptions of Theorem 1.1 suppose that $f_{0}$ is a germ of a local biholomorphism at $x$ with $f_{0}(M) \subset M$. Then there exists a neighborhood $U(x) \in \mathbb{C}^{n}$ such that all germs of local biholomorphisms $f$ with $f(M) \subset M$ and $j_{x}^{k} f$ sufficiently close to $j_{x}^{k} f_{0}$ extend biholomorphically to $U(x)$.

Following Tanaka [14] we call a map $\varphi: M \rightarrow M^{\prime}$ pseudo-conformal, if it extends to a holomorphic map between some neighborhoods of $M$ and $M^{\prime}$ respectively. One obtains the following global version of Corollary 1.1 (see the end of the section for the proof).

Corollary 1.3. Let $M$ be a compact $C R$ submanifold of a complex manifold $X$ which in addition satisfies the assumptions of Theorem 1.1. Then the group of all pseudo-conformal automorphisms of $M$ is a Lie group.

In fact, all of these corollaries are proved here in the more general situation, where $M \subset \mathbb{C}^{n}$ and $M^{\prime} \subset \mathbb{C}^{n^{\prime}}$ are generic real-analytic CR manifolds of arbitrary $\mathrm{CR}$ dimension and codimension. In particular, the unique determinacy by $k$-jets, i.e. the injectivity of the $k$-jet evaluation $f \mapsto j_{x}^{k} f$, is also shown.

In this paper $f$ always denotes a germ of a holomorphic map between open subsets of $\mathbb{C}^{n}$ and $\mathbb{C}^{n^{\prime}}$ respectively. The condition " $f$ is biholomorphic" (see $[1],[2])$ is relaxed to

$$
d f_{x}\left(T_{x}^{c} M\right)=T_{x^{\prime}}^{c} M^{\prime}
$$

where $f(x)=x^{\prime}$ and $T_{x}^{c} M:=T_{x} M \cap i T_{x} M \subset T_{x} \mathbb{C}^{n}$ is the complex tangent space. 
The following simple example shows that even if both $\mathrm{CR}$ dimensions and codimensions of $M$ and $M^{\prime}$ are equal, the case when (4) is satisfied but $f$ is not biholomorphic is also of interest.

Example 1.1. Let $\varphi$ be strongly plurisubharmonic function in a neighborhood of $0 \in \mathbb{C}^{3}$ satisfying

$$
\varphi(0)=0, \quad \frac{\partial \varphi}{\partial z_{3}}(0) \neq 0
$$

Define $M$ and $M^{\prime}$ in $\mathbb{C}^{4}$ :

$$
\begin{gathered}
M:=\left\{\left(z_{1}, z_{2}, z_{3}, z_{4}\right): \varphi\left(z_{1}, z_{2}, z_{3}\right)=\varphi\left(2 z_{1}, z_{2}, z_{4}\right)=0\right\}, \\
M^{\prime}:=\left\{\left(z_{1}, z_{2}, z_{3}, z_{4}\right): \varphi\left(z_{1}, z_{2}, z_{3}\right)=\operatorname{Re} z_{4}=0\right\} .
\end{gathered}
$$

One has

$$
\operatorname{dim}_{\mathrm{CR}} M=\operatorname{dim}_{\mathrm{CR}} M^{\prime}=2, \quad \operatorname{codim}_{\mathrm{CR}} M=\operatorname{codim}_{\mathrm{CR}} M^{\prime}=2 .
$$

The $\left(\mathbb{C}^{2}\right.$-valued) Levi forms are given by the second order derivatives $\partial^{2} \varphi / \partial z_{i} \partial \bar{z}_{j}$. Simple calculation shows that the Levi form of $M$ (resp. $M^{\prime}$ ) is surjective (resp. nondegenerate). By Proposition 2.1 and Lemma 2.1, $M$ and $M^{\prime}$ satisfy conditions of Theorem 1.2 below. The map $f$ given by

$$
f\left(z_{1}, z_{2}, z_{3}, z_{4}\right):=\left(z_{1}, z_{2}, z_{3}, 0\right)
$$

satisfies $f(M) \subset M^{\prime}$ and condition (4) but is not a diffeomorphism between $M$ and $M^{\prime}$.

On the other hand, the condition (4) cannot be removed as the following elementary example shows.

Example 1.2. Define

$$
M:=\left\{\left|z_{1}\right|^{2}+\left|z_{2}\right|^{2}-\left|z_{3}\right|^{2}=1\right\} \subset \mathbb{C}^{3} .
$$

Then $M$ contains the complex line

$$
L=\left\{z_{1}=1, z_{2}=z_{3}\right\}
$$

Therefore every holomorphic map $f: \mathbb{C}^{3} \rightarrow L$ satisfies $f(M) \subset M$. Although the Levi form of $M$ is nondegenerate and surjective, the $k$-jet evaluation $f \mapsto j_{x}^{k} f$ is not injective for no integer $k$. 
As noted above, the nondegeneracy and surjectivity of the Levi form are only sufficient conditions. The optimal conditions which are necessary in many cases are given in [2] for the case $f$ is biholomorphic. Here we reformulate them in a form suitable for our purposes.

We first recall the notion of the Segre varieties associated to a generic realanalytic CR submanifold $M \subset \mathbb{C}^{n}$. Let $x \in M$ be arbitrary and let $M$ be defined near $x$ by the real-analytic equations

$$
\rho_{1}(z, \bar{z})=\cdots=\rho_{d}(z, \bar{z})=0, \quad \partial \rho_{1} \wedge \cdots \wedge \partial \rho_{d} \neq 0 .
$$

The complexification $\mathcal{M} \subset \mathbb{C}^{n} \times \overline{\mathbb{C}^{n}}$ is defined by

$$
\mathcal{M}:=\left\{(z, \bar{w}) \in U(x) \times U(\bar{x}): \rho_{1}(z, \bar{w})=\cdots=\rho_{d}(z, \bar{w})=0\right\},
$$

where $U(x) \subset \mathbb{C}^{n}$ and $U(\bar{x}) \subset \overline{\mathbb{C}^{n}}$ are sufficiently small neighborhoods. As a germ at $(x, \bar{x})$ of a complex-analytic subset, $\mathcal{M}$ is independent of the choice of the defining equations (12). By fixing the coordinate $\bar{w}$, we obtain the Segre variety

$$
Q_{w}:=\{z \in U(x):(z, \bar{w}) \in \mathcal{M}\} .
$$

Segre varieties were introduced by Segre [13] and play an important role in the reflection principle (see e.g. $[12,10,9,16,17]$ ). For $z, w$ close to $x$, it follows that $Q_{w}$ is a complex manifold of dimension $n-d=\operatorname{dim}_{\mathrm{CR}} M$. The following symmetry property is a direct corollary of the invariance of $\mathcal{M}$ under the involution $(z, \bar{w}) \mapsto(w, \bar{z})$ :

$$
z \in Q_{w} \Longleftrightarrow w \in Q_{z}
$$

For arbitrary $x \in \mathbb{C}^{n}$, denote by $J_{x}^{k, d}\left(\mathbb{C}^{n}\right)$ the space of $k$-jets at $x$ of $d$ codimensional complex submanifolds $V \subset \mathbb{C}^{n}$ with $x \in V$ and by $j_{x}^{k}(V) \in$ $J_{x}^{k, d}\left(\mathbb{C}^{n}\right)$ the $k$-jet of $V$. The following is another form of the definition of a $k$-nondegenerate manifold given in [1].

Definition 1.3. A generic real-analytic CR manifold $M \subset \mathbb{C}^{n}$ is called $k$ nondegenerate at $x \in M(k \geq 1)$, if the (antiholomorphic) map

$$
\varphi^{k}: Q_{x} \rightarrow J_{x}^{k, d}\left(\mathbb{C}^{n}\right), w \mapsto j_{x}^{k}\left(Q_{w}\right),
$$

is of the rank $n-d=\operatorname{dim} Q_{x}$ at $x . M$ is called finitely nondegenerate, if it is $k$-nondegenerate for some $k$.

A manifold $M$ is called essentially finite at $x$ if the germ of $Q_{x}$ at $x$ is different from germs of $Q_{w}$ at $x$ for all $w \in Q_{x} \backslash\{x\}$ sufficiently close to $x$. This notion was introduced in [4] and earlier implicitly discussed in [8]. By Proposition 1.3.1 in [1], if $M$ is essentially finite at $x$, there exists the so-called Levi number $l(M)$. Then $M$ is $l(M)$-nondegenerate at all points $p \in M \backslash W$, where $W \subset M$ 
is a proper real-analytic subset. Clearly the $k$-nondegeneracy guarantees the $k^{\prime}$-nondegeneracy for all $k^{\prime} \geq k$.

The second important notion is the minimality condition introduced by Tumanov in [15]:

Definition 1.4. A CR manifold $M$ is called minimal at $x \in M$, if there does not exist a proper CR submanifold $N \subset M$ with $x \in N$ such that $\operatorname{dim}_{\mathrm{CR}} N=$ $\operatorname{dim}_{\mathrm{CR}} M$.

Tumanov [15] shows that, if $M$ is minimal at $x$, all CR functions on $M$ extend holomorphically to a wedge with the edge $M$.

Definition 1.5. We call a germ $f$ admissible if it satisfies the following conditions:

$$
f(M) \subset M^{\prime}, \quad d f\left(T_{x}^{c} M\right)=T_{f(x)}^{c} M^{\prime} .
$$

Theorem 1.2. Suppose that $M^{\prime}$ is $r$-nondegenerate, $M$ is minimal at $x$ and $k:=2 r\left(1+\operatorname{codim}_{\mathrm{CR}} M\right)$. Let $S$ be the set of all admissible germs $f$ at $x$. Then the germs in $S$ depend analytically on their $k$-jets at $x$.

A different proof of Theorem 1.2 in the case of hypersurfaces $\left(\operatorname{codim}_{\mathrm{CR}} M=\right.$ $\left.\operatorname{codim}_{\mathrm{CR}} M^{\prime}=1\right)$ with a sharper estimate for the jet order $(2 r$-jets instead of $4 r$-jets) has been obtained recently by Baouendi, Ebenfelt and Rothschild [3].

Remark. The case, where one of the manifolds $M, M^{\prime}$ is not generic, can be reduced to the generic case. Every real-analytic CR submanifold $M \subset \mathbb{C}^{n}$ is generic in the so-called intrinsic complexification $V \subset \mathbb{C}^{n}$ defined to be the minimal (in the sense of germs) complex-analytic subvariety which contains $M$. If $M$ is CR, $V$ is smooth. After a change of local coordinates $V$ becomes open in $\mathbb{C}^{m} \subset \mathbb{C}^{n}$ near $x \in M$. The condition $f(M) \subset M^{\prime}$ automatically implies $f(V) \subset V^{\prime}$. If $M^{\prime}$ is not generic, we can therefore replace $\mathbb{C}^{n^{\prime}}$ with $V^{\prime}$. If $M$ is not generic, Theorem 1.2 yields a parametrization (3) for the restricted admissible germs $f: V \rightarrow \mathbb{C}^{n^{\prime}}$, whereas outside $V$ the germs can be chosen arbitrary and cannot be determined by their $s$-jets even for $s$ arbitrary large.

As a corollary, we obtain the following description of the space of admissible germs as a (locally closed) real-analytic subset of $J_{x}^{k}\left(\mathbb{C}^{n}, \mathbb{C}^{n^{\prime}}\right)$.

Corollary 1.4. Let $k, r, x, M, M^{\prime}, S$ satisfy the conditions of Theorem 1.2.

(1) Then the set $A \subset J_{x}^{k}\left(\mathbb{C}^{n}, \mathbb{C}^{n^{\prime}}\right)$ of the $k$-jets of all $f \in S$ is a locally closed real-analytic subset.

(2) There exists a neighborhood $U$ of $A \times\{x\} \subset A \times \mathbb{C}^{n}$ and a (unique) real-analytic map

$$
G: U \rightarrow \mathbb{C}^{n^{\prime}}
$$


which is holomorphic in the $\mathbb{C}^{n}$-factor and is such that every $f \in S$ satisfies

$$
f(w)=G\left(j_{x}^{k} f, w\right) .
$$

Proof. By Theorem 1.2, the germs in $S$ depend analytically on their $k$-jets. Let $M, M^{\prime}$ be closed in some open subsets

$$
U(M) \subset \mathbb{C}^{n}, \quad U\left(M^{\prime}\right) \subset \mathbb{C}^{n^{\prime}}
$$

respectively. For every $f_{0} \in S$, Theorem 1.2 yields the local parametrization (3). Without loss of generality, $U(x) \subset U(M)$ and

$$
F\left(U\left(j_{x}^{k} f_{0}\right) \times U(x)\right) \subset U\left(M^{\prime}\right) .
$$

Then the above set $A \subset J_{x}^{k}\left(\mathbb{C}^{n}, \mathbb{C}^{n^{\prime}}\right)$ is locally defined by

$$
\begin{aligned}
A \cap U\left(j_{x}^{k} f_{0}\right):=\left\{j \in U\left(j_{x}^{k} f_{0}\right):\right. & \\
& \left.F(j, w) \subset M^{\prime} \text { for all } w \in M \cap U(x), \quad j=j_{x}^{k} F(j, \cdot)\right\} .
\end{aligned}
$$

It follows from the real-analyticity of $M$ and $M^{\prime}$ that $A$ is locally defined by finitely many real-analytic equations, i.e. it is a real-analytic subset. The restrictions of $F$ 's to $A \times U(x)$ for different $f_{0}$ 's glue together to a well-defined real-analytic map $G$ satisfying the required properties.

Proof.[Corollary 1.1] By Corollary 1.4, the set $A \subset J_{x}^{k}\left(\mathbb{C}^{n}, \mathbb{C}^{n^{\prime}}\right)$ of the $k$-jets $(k:=2(d+1) r)$ of all admissible germs is real-analytic. Denote by $A_{0} \subset A$ the subset of the $k$-jets fixing $x$. Clearly $A_{0}$ is also real-analytic and locally closed. Since $A_{0}$ is also closed under the composition of the jets, it is a closed Lie subgroup of the Lie group of all $k$-jets fixing $x$.

Proof.[Corollary 1.3] By Theorem 1.2 and the definition of analytic dependence on $k$-jets, there exists a finite open covering of $M$ with coordinate neighborhoods $U\left(x_{i}\right)$ with parametrizations

$$
F_{i}: U\left(j_{x_{i}}^{k} f_{0}\right) \times U\left(x_{i}\right) \rightarrow \mathbb{C}^{n},
$$

where $f_{0}:=\mathrm{id} \in \operatorname{Aut}(M)$. An automorphism $f \in \operatorname{Aut}(M)$ in a neighborhood of id is therefore uniquely determined by finitely many parameters

$$
p_{i}=j_{x_{i}}^{k} f, \quad q_{i}=j_{x_{i}}^{k} f^{-1} .
$$

Set $f_{i}:=F_{i}\left(p_{i}, \cdot\right), g_{i}:=F_{i}\left(q_{i}, \cdot\right)$. A tuple $\left(p_{1}, \ldots, p_{m}, q_{1}, \ldots, q_{m}\right)$ determines an automorphism of $M$ if and only if the following is satisfied:

(1) $f_{i}=f_{j}, g_{i}=g_{j}$ on $U\left(x_{i}\right) \cap U\left(x_{j}\right)$ for all $i, j$;

(2) $f_{i}(M) \subset M, g_{i}(M) \subset M$ for all $i$;

(3) The glued maps $f_{p}$ and $g_{q}$ satisfy $f_{p} \circ g_{q}=g_{q} \circ f_{p}=\mathrm{id}, j_{x_{i}}^{k} f_{p}=p_{i}$, $j_{x_{i}}^{k} g_{q}=q_{i}$. 
Conditions (1.),(2.),(3.) define an analytic subset of the parameter space of $p_{i}$ 's and $q_{i}$ 's. In these coordinates the group operation is given by

$$
\left((p, q),\left(p^{\prime}, q^{\prime}\right)\right) \mapsto\left(j_{x_{i}}^{k}\left(f_{p^{\prime}} \circ f_{p}\right), j_{x_{i}}^{k}\left(g_{q} \circ g_{q^{\prime}}\right)\right)
$$

and is therefore real-analytic. Hence $\operatorname{Aut}(M)$ is a Lie group.

\section{The Levi form and nondegeneracy conditions}

Let $M \subset \mathbb{C}^{n}$ be a CR submanifold and $x \in M$ an arbitrary point. Recall that a (1,0)-vector field on $M$ is a vector field $X$ in $T^{c} M \otimes_{\mathbb{R}} \mathbb{C}$ such that $J X=i X$, where $J: T^{c} M \otimes_{\mathbb{R}} \mathbb{C} \rightarrow T^{c} M \otimes_{\mathbb{R}} \mathbb{C}$ is the complexification of the CR structure $J: T^{c} M \rightarrow T^{c} M$ and $i X$ is the multiplication by $i$ in the component $\mathbb{C}$ of the tensor product.

The Levi form of $M$ at $x$ is the hermitian (vector-valued) form

$$
L: T_{x}^{1,0} M \times T_{x}^{1,0} M \rightarrow\left(T_{x} M / T_{x}^{c} M\right) \otimes_{\mathbb{R}} \mathbb{C},
$$

given by

$$
L(X, Y):=\frac{1}{2 i} \pi([X, \bar{Y}])
$$

where $X, Y$ are $(1,0)$-vector fields on $M$ and

$$
\pi: T_{x} M \otimes_{\mathbb{R}} \mathbb{C} \rightarrow\left(T_{x} M / T_{x}^{c} M\right) \otimes_{\mathbb{R}} \mathbb{C}
$$

is the canonical projection. Notice that $L(X, Y)(x)$ depends only on $X(x), Y(x)$.

Remark. If $M \subset \mathbb{C}^{n}$ is a hypersurface defined by $M=\{\varphi=0\}$ with $d \varphi \neq 0$, the standard Levi form of $\varphi$ coincides with the evaluation of $\partial \varphi$ on $L(X, Y)$.

The Levi form is a first order holomorphic invariant of $M$ whereas the conditions in Theorem 1.2 are of possibly higher order. However, we obtain the following

Proposition 2.1. A generic real-analytic $C R$ manifold $M \subset \mathbb{C}^{n}$ is 1-nondegenerate at $x$ if and only if the Levi form at $x$ is nondegenerate.

Proof. Let $M$ be locally defined near $x$ by the real-analytic equations

$$
\rho_{1}(z, \bar{z})=\cdots=\rho_{d}(z, \bar{z})=0,
$$

where $\partial \rho_{1} \wedge \cdots \wedge \partial \rho_{d} \neq 0$.

The differentials $\partial \rho_{j}$ vanish on $T^{c} M \otimes_{\mathbb{R}} \mathbb{C}$. Hence, for a (1,0)-vector field $X \subset T^{1,0} M$,

$$
\partial \rho_{j}(X)=\partial \rho_{j}(\bar{X})=0 .
$$

Denote by $X \psi$ the derivative of $\psi$ along $X$.

Applying the formula

$$
d \omega\left(X_{1}, X_{2}\right)=X_{1} \omega\left(X_{2}\right)-X_{2} \omega\left(X_{1}\right)-\omega\left(\left[X_{1}, X_{2}\right]\right)
$$


for $\omega=\partial \rho_{j}, X_{1}=X, X_{2}=\bar{Y}$, we obtain

$$
\bar{\partial} \partial \rho_{j}(X, \bar{Y})=-\partial \rho_{j}([X, \bar{Y}]) .
$$

The Segre varieties $Q_{w}$ are given by

$$
\rho_{1}(z, \bar{w})=\cdots=\rho_{d}(z, \bar{w})=0
$$

and their 1 -jets $j_{x}^{1} Q_{w}$ by the linear equations

$$
\partial \rho_{1}(z, \bar{w})=\cdots=\partial \rho_{s}(z, \bar{w})=0 .
$$

Let $e_{1}(z, \bar{w}), \ldots, e_{d}(z, \bar{w})$ be a collection of $(1,0)$-vector fields on the complexification $\mathcal{M}$ which is a basis of $T_{z} Q_{w}$ at every point $(z, \bar{w}) \in \mathcal{M}$ close to $(x, \bar{x})$. Let $e_{1}^{\prime}, \ldots, e_{d}^{\prime}$ be a similar collection of $(1,0)$-vector fields in the $w$-direction, i.e. pointwise a basis of $T_{\bar{w}} \overline{Q_{\bar{z}}}$. The rank of the map $\varphi^{1}$ in Definition 1.3 is the same as the rank of the matrix with the rows

$$
\bar{\partial} \partial \rho_{j}\left(e_{k}, \bar{e}_{l}^{\prime}\right) \text { with } l \text { fixed . }
$$

By (31), this matrix has the rows

$$
-\partial \rho_{j}\left(\left[e_{k}, \bar{e}_{l}^{\prime}\right]\right) \text { with } l \text { fixed } .
$$

Since $\partial \rho_{1} \wedge \cdots \wedge \partial \rho_{d} \neq 0$, the tuple $\left(\partial \rho_{1}, \ldots, \partial \rho_{d}\right)$ defines an isomorphism between $\left(T M / T^{c} M\right) \otimes_{\mathbb{R}} \mathbb{C}$ and $\mathbb{C}^{d}$. Hence the matrix with the rows (35) has the same rank as the matrix with the rows

$$
L\left(e_{k}, e_{l}\right) \text { with } l \text { fixed } .
$$

This rank equals to $n-d$ if and only if the Levi form of $M$ is nondegenerate.

The minimality condition (Definition 1.4) involves high order commutators and therefore cannot be formulated in terms of the Levi form. However, one obviously has the following sufficient condition.

Lemma 2.1. Suppose that the Levi form of $M$ at $x$ is surjective onto $\left(T_{x} M / T_{x}^{c} M\right) \otimes_{\mathbb{R}} \mathbb{C}$. Then $M$ is minimal at $x$.

Now we can formulate special cases of Theorem 1.2 and Corollary 1.4 under Levi form conditions.

Theorem 2.1. Suppose that the Levi form of $M^{\prime}$ is everywhere nondegenerate, the Levi form of $M$ is surjective at $x$ and $d=\operatorname{codim}_{\mathrm{CR}} M$. Then the conclusions of Theorem 1.2 and Corollary 1.4 hold. 


\section{Local parametrization of jets of holomorphic maps}

One of the technical tools for proving Theorem 1.2 is the following connection between the $k$-jets at $z$ and the $(k+r)$-jets at $\bar{w}$. For simplicity, we write for a subset $U(j) \subset J^{k+r}\left(\mathbb{C}^{n}, \mathbb{C}^{n^{\prime}}\right)$,

$$
U_{z}(j):=U(j) \cap J_{z}^{k+r}\left(\mathbb{C}^{n}, \mathbb{C}^{n^{\prime}}\right) .
$$

Proposition 3.1. Suppose that $M^{\prime}$ is $r$-nondegenerate at $x^{\prime}$ and $f_{0}$ is an admissible germ at $x \in M, x^{\prime}=f_{0}(x)$. Then, for every integer $k$ there exist neighborhoods $U\left(j_{x}^{k+r} f_{0}\right) \subset J^{k+r}\left(\mathbb{C}^{n}, \mathbb{C}^{n^{\prime}}\right), U(x, \bar{x}) \subset \mathcal{M}$ and for every $(z, \bar{w}) \in U(x, \bar{x})$ a holomorphic map $F_{(z, \bar{w})}^{k}: U_{z}\left(j_{x}^{k+r} f_{0}\right) \rightarrow J_{\bar{w}}^{k}\left(\overline{\mathbb{C}^{n}}, \overline{\mathbb{C}^{n^{\prime}}}\right)$, such that for all admissible germs $f$ at $x$ with $j_{x}^{k+r} f \in U\left(j_{x}^{k+r} f_{0}\right)$,

$$
j_{\bar{w}}^{k} \bar{f}=F_{(z, \bar{w})}^{k}\left(j_{z}^{k+r} f\right),
$$

where $(z, \bar{w}) \subset \mathcal{M}$ is sufficiently close to $(x, \bar{x})$. Moreover, the map $F_{(z, \bar{w})}^{k}$ depends holomorphically on $(z, \bar{w}) \in U(x, \bar{x})$.

A different proof of Proposition 3.1 is given in [1] (Assertion 3.3.1) and [2] (Proposition 2.2).

Proof. Let $(z, \bar{w}) \in \mathcal{M}$ be close to $(x, \bar{x})$. By the construction (see (12),(13),(14)), the Segre variety $Q_{w}$ is smooth at $z$ and $d:=\operatorname{dim}_{z} Q_{w}$ is constant. We choose local coordinates $z=\left(z_{1}, z_{2}\right) \in \mathbb{C}^{d} \times \mathbb{C}^{n-d}$ near $x$ such that the Segre variety $Q_{w}$ has the form of the graph:

$$
Q_{w}=\left\{\left(z_{1}, \varphi_{w}\left(z_{1}\right)\right): z_{1} \in U\left(x_{1}\right)\right\}, \quad x=\left(x_{1}, x_{2}\right),
$$

where $U\left(x_{1}\right) \subset \mathbb{C}^{n}$ is an open neighborhood and $\varphi_{w}: U\left(x_{1}\right) \rightarrow \mathbb{C}^{n-d}$ is holomorphic. The map $\varphi_{w}$ is uniquely defined and depends holomorphically on $\bar{w}$ in some neighborhood $U(\bar{x}) \subset \overline{\mathbb{C}^{n}}$. Denote by $j_{z}^{r} Q$ the holomorphic $r$-jet evaluation

$$
j_{z}^{r} Q: U(\bar{x}) \rightarrow J_{z_{1}}^{r}\left(\mathbb{C}^{d}, \mathbb{C}^{n-d}\right), \quad \bar{w} \mapsto j_{z_{1}}^{r} \varphi_{w} .
$$

A similar evaluation is obtained for $\left(z^{\prime}, \bar{w}^{\prime}\right) \in \mathcal{M}^{\prime}$ sufficiently close to $\left(x^{\prime}, \bar{x}^{\prime}\right)$ :

$$
j_{z^{\prime}}^{r} Q^{\prime}: U\left(\bar{x}^{\prime}\right) \rightarrow J_{z_{1}^{\prime}}^{r}\left(\mathbb{C}^{d^{\prime}}, \mathbb{C}^{n^{\prime}-d^{\prime}}\right), \quad \bar{w}^{\prime} \mapsto j_{z_{1}^{\prime}}^{r} \varphi_{w^{\prime}}^{\prime}
$$

We claim that (41) is an immersion. Since $M^{\prime}$ is $r$-nondegenerate at $x^{\prime}=$ $\left(x_{1}^{\prime}, x_{2}^{\prime}\right), j_{x}^{r} Q^{\prime}$ restricted to the Segre variety $Q_{x^{\prime}}^{\prime}$ is an immersion. By the construction, the 0-jet evaluation $j_{x^{\prime}}^{0} Q^{\prime}=x_{2}^{\prime}$ is constant on $Q_{x^{\prime}}^{\prime}$. On the other hand, the restriction of $j_{x^{\prime}}^{0} Q^{\prime}$ to the transversal direction, i.e. $\left\{x_{1}^{\prime}\right\} \times \mathbb{C}^{n^{\prime}-d^{\prime}}$, is also an immersion. Indeed, since $M^{\prime} \cap\left(\left\{x_{1}^{\prime}\right\} \times \mathbb{C}^{n^{\prime}-d^{\prime}}\right)$ is totally real in $\left\{x_{1}^{\prime}\right\} \times \mathbb{C}^{n^{\prime}-d^{\prime}}$, it is locally biholomorphically equivalent to $\mathbb{R}^{n^{\prime}-d^{\prime}}$. For $\mathbb{R}^{s} \subset \mathbb{C}^{s}$, the immersion property of $j_{x^{\prime}}^{0} Q^{\prime}$ can be directly verified.

Hence the total map (41) splits into $j_{x^{\prime}}^{0} Q^{\prime}$ which is an immersion on $\left\{x_{1}^{\prime}\right\} \times$ $\mathbb{C}^{n^{\prime}-d^{\prime}}$ and constant on $Q_{x^{\prime}}^{\prime}$ and the remainder which is an immersion on $Q_{x^{\prime}}^{\prime}$. 
Therefore (41) is an immersion for $z$ close to $x$ and locally there exists a left inverse map $\left(j_{z^{\prime}}^{r} Q^{\prime}\right)^{-1}$ which satisfies $\left(j_{z^{\prime}}^{r} Q^{\prime}\right)^{-1} \circ\left(j_{z^{\prime}}^{r} Q^{\prime}\right)=$ id.

Let $f_{0}$ be an admissible germ. Complexifying the condition $f_{0}(M) \subset M^{\prime}$ we obtain $\left(f_{0}, \bar{f}_{0}\right)(\mathcal{M}) \subset \mathcal{M}^{\prime}$, which means

$$
f_{0}\left(Q_{w}\right) \subset Q_{f_{0}(w)}^{\prime} .
$$

Since $\operatorname{dim} Q_{w}$ is constant, the second condition $d f\left(T_{x}^{c} M\right)=T_{f(x)}^{c} M^{\prime}$ implies

$$
\begin{aligned}
\operatorname{dim} f_{0}\left(Q_{w}\right)=\operatorname{dim} d f_{0}\left(T_{x} Q_{x}\right)= & \operatorname{dim} d f_{0}\left(T^{c} M\right) \\
& =\operatorname{dim} T^{c} M^{\prime}=\operatorname{dim} Q_{x^{\prime}}=\operatorname{dim} Q_{f_{0}(w)}^{\prime} .
\end{aligned}
$$

Along with (42) this yields

$$
f_{0}\left(Q_{w}\right)=Q_{f_{0}(w)}^{\prime}, \quad d f_{0}\left(T_{z} Q_{w}\right)=T_{z^{\prime}} Q_{w^{\prime}}^{\prime} .
$$

After going to the $k$-jet evaluations we obtain the following commutative diagram

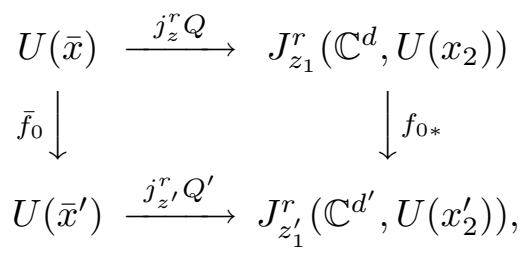

where $f_{0 *}$ is the corresponding map on the jet level defined as follows.

By (44), there exists a local splitting of the source space $\mathbb{C}^{d}=\mathbb{C}^{d^{\prime}} \times \mathbb{C}^{d-d^{\prime}}$ near $x_{1}=\left(x_{11}, x_{12}\right)$ such that the restriction

$$
f_{0}: Q_{w} \cap\left\{z_{12}=x_{12}\right\} \rightarrow Q_{w^{\prime}}^{\prime}
$$

is locally biholomorphic for all $(z, \bar{w}) \in \mathcal{M}$ close to $(x, \bar{x})$.

We write $f_{01}: U(x) \rightarrow \mathbb{C}^{d^{\prime}}, f_{02}: U(x) \rightarrow \mathbb{C}^{n^{\prime}-d^{\prime}}$ for the components of $f_{0}$ (here $\mathbb{C}^{d^{\prime}}$ and $\mathbb{C}^{n^{\prime}-d^{\prime}}$ are in the target space). Since (46) is locally biholomorphic, the map

$$
\tilde{f}_{0}: \mathbb{C}^{d^{\prime}} \rightarrow \mathbb{C}^{d^{\prime}}, \quad z_{11} \mapsto f_{01}\left(z_{11}, x_{12}, \varphi_{\bar{w}}\left(z_{11}, x_{12}\right)\right)
$$

is also locally biholomorphic.

Let $\tilde{f}_{0}^{-1}$ be the local inverse. By (39) and (44)

$$
\varphi_{\bar{w}^{\prime}}^{\prime}\left(z_{1}^{\prime}\right)=f_{02}\left(\tilde{f}_{0}^{-1}\left(z_{1}^{\prime}\right), x_{12}, \varphi_{\bar{w}}\left(\tilde{f}_{0}^{-1}\left(z_{1}^{\prime}\right), x_{12}\right)\right)
$$

and hence, passing to the $r$-jets,

$$
j_{z_{1}^{\prime}}^{r} \varphi_{\bar{w}^{\prime}}^{\prime}=\left(j_{z}^{r} f_{02}\right)\left(\left(j_{z_{11}}^{r} \tilde{f}_{0}\right)^{-1}, x_{12},\left(j_{z_{1}}^{r} \varphi_{\bar{w}}\right)\left(\left(j_{z_{11}}^{r} \tilde{f}_{0}\right)^{-1}, x_{12}\right)\right),
$$

where by (47),

$$
j_{z_{11}}^{r} \tilde{f}_{0}=\left(j_{z}^{r} f_{01}\right)\left(z_{11}, x_{12},\left(j_{z_{1}}^{r} \varphi_{\bar{w}}\right)\left(z_{11}, x_{12}\right)\right)
$$


with obvious notation.

The formulae $(49,50)$ can be written together in the form

$$
j_{z_{1}^{\prime}}^{r} \varphi_{\bar{w}^{\prime}}^{\prime}=\Phi\left(j_{z}^{r} f_{0}, j_{z_{1}}^{r} \varphi_{\bar{w}}\right)
$$

where

$$
\Phi: U_{z}\left(j_{x}^{r} f_{0}\right) \times U_{z_{1}}\left(j_{x_{1}}^{r} \varphi_{\bar{x}}\right) \rightarrow J_{z_{1}^{\prime}}^{r}\left(\mathbb{C}^{d^{\prime}}, U\left(x_{2}^{\prime}\right)\right)
$$

is a holomorphic family of maps and $U\left(j_{x}^{r} f_{0}\right) \subset J^{r}\left(\mathbb{C}^{n}, \mathbb{C}^{n^{\prime}}\right), U\left(j_{x_{1}}^{r} \varphi_{\bar{x}}\right) \subset$ $J^{r}\left(\mathbb{C}^{d}, \mathbb{C}^{n-d}\right)$ are sufficiently small neighborhoods.

Define $f_{0 *}$ as in (45) by

$$
f_{0 *}:=\Phi\left(j_{z}^{r} f_{0}, \cdot\right) .
$$

By (51), the diagram (45) is commutative.

The commutativity means that

$$
\left(j_{z^{\prime}}^{r} Q^{\prime}\right) \circ \bar{f}_{0}=f_{0 *} \circ\left(j_{z}^{r} Q\right) .
$$

Applying the left inverse $\left(j_{z^{\prime}}^{r} Q^{\prime}\right)^{-1}$ to both sides we obtain

$$
\bar{f}_{0}=\left(j_{z^{\prime}}^{r} Q^{\prime}\right)^{-1} \circ f_{0 *} \circ\left(j_{z}^{r} Q\right)
$$

and, passing to the $k$-jets,

$$
j_{\bar{w}}^{k} \bar{f}_{0}=j_{g^{\prime}}^{k}\left(j_{z^{\prime}}^{r} Q^{\prime}\right)^{-1} \circ j_{g}^{k} f_{0 *} \circ j_{\bar{w}}^{k}\left(j_{z}^{r} Q\right),
$$

where $g:=\left(j_{z}^{r} Q\right)(\bar{w})$ and $g^{\prime}:=j_{g}^{k} f_{0 *}(g)$.

The $k$-jet $j_{g}^{k} f_{0 *}$ can be calculated from (53):

$$
j_{g}^{k} f_{0 *}:=\left(j_{s}^{k} \Phi\right)\left(j_{z}^{k+r} f_{0}, \cdot\right), \quad s:=\left(j_{z}^{r} f_{0}, g\right) .
$$

We write (56) and (57) together in the form

$$
j_{\bar{w}}^{k} \bar{f}_{0}=F_{z, \bar{w}}^{k}\left(j_{z}^{k+r} f_{0}\right),
$$

where

$$
F_{z, \bar{w}}^{k}: U_{z}\left(j_{x}^{k+r} f_{0}\right) \rightarrow J_{\bar{w}}^{k}\left(\overline{\mathbb{C}^{n}}, \overline{\mathbb{C}^{n^{\prime}}}\right)
$$

is a holomorphic family of maps and $U\left(j_{x}^{k+r} f_{0}\right) \subset J^{k+r}\left(\mathbb{C}^{n}, \mathbb{C}^{n^{\prime}}\right)$ a sufficiently small open neighborhood.

Now we notice that $f_{0}$ was an arbitrary admissible germ. Let $f$ be another one with $j_{x}^{k+r} f \in U\left(j_{x}^{k+r} f_{0}\right)$. Then for $(z, \bar{w}) \in \mathcal{M}$ sufficiently close to $(x, \bar{x})$, (48)-(58) remain valid if we replace $f_{0}$ with $f$. In particular, the proposition follows from (58) with $f_{0}$ replaced by $f$. 


\section{Proof of Theorem 1.2}

Given $j_{z}^{k+r}$ for $z \in \mathbb{C}^{n}$ fixed, (38) yields the $k$-jet $j_{w}^{k} f$, in particular $f$ itself $\left(f(w)=j_{w}^{0} f\right)$. However, the point $w$ is not arbitrary but must satisfy

$$
(z, \bar{w}) \in \mathcal{M} \text {, i.e. } w \in Q_{z} .
$$

Our next goal will be to obtain a similar formula which yields $f$ at least on an open subset of $\mathbb{C}^{n}$. The idea here is to iterate the construction of the previous paragraph.

4.1. Iterated complexification. The iterated complexification $\mathcal{M}^{s}$ of $\mathcal{M}$ is defined by

$$
\begin{aligned}
\mathcal{M}^{2 l-1} & :=\left\{\left(z_{1}, \bar{w}_{1}, \ldots, z_{l}, \bar{w}_{l}\right) \in \mathbb{C}^{n} \times \overline{\mathbb{C}^{n}} \times \cdots \times \mathbb{C}^{n} \times \overline{\mathbb{C}^{n}}:\right. \\
& \left.\left(z_{1}, \bar{w}_{1}\right) \in \mathcal{M},\left(w_{1}, \bar{z}_{2}\right) \in \mathcal{M}, \ldots,\left(w_{l-1}, \bar{z}_{l}\right) \in \mathcal{M},\left(z_{l}, \bar{w}_{l}\right) \in \mathcal{M}\right\},
\end{aligned}
$$

if $s=2 l-1$ is odd and by

$$
\begin{aligned}
\mathcal{M}^{2 l}:= & \left\{\left(z_{1}, \bar{w}_{1}, \ldots, z_{l}, \bar{w}_{l}, z_{l+1}\right) \in \mathbb{C}^{n} \times \overline{\mathbb{C}^{n}} \times \cdots \times \mathbb{C}^{n} \times \overline{\mathbb{C}^{n}} \times \mathbb{C}^{n}:\right. \\
& \left.\left(z_{1}, \bar{w}_{1}\right) \in \mathcal{M},\left(w_{1}, \bar{z}_{2}\right) \in \mathcal{M}, \ldots,\left(z_{l}, \bar{w}_{l}\right) \in \mathcal{M},\left(w_{l}, \bar{z}_{l+1}\right) \in \mathcal{M}\right\}
\end{aligned}
$$

if $s=2 l$ is even.

For simplicity we restrict the proof of Theorem 1.2 in the sequel to the case (61) of odd iterates. The case (62) of even iterates is completely analogous. We write

$$
\mathcal{M}_{z}^{s}:=\mathcal{M}^{s} \cap\left\{z_{1}=z\right\} .
$$

Lemma 4.1. The analytic subset $\mathcal{M}^{s} \subset \mathbb{C}^{N}$ is smooth at $(x, \ldots, \bar{x})$ and the projection on the first copy of $\mathbb{C}^{n}$ is of rank $n$ at this point. $\mathcal{M}_{x}^{s}$ is also smooth at $(x, \ldots, \bar{x})$.

Proof. By the construction of the Segre varieties (see (12),(13),(14)), there exists a local splitting $\overline{\mathbb{C}^{n}(w)}=\overline{\mathbb{C}^{d}\left(w^{1}\right)} \times \overline{\mathbb{C}^{n-d}\left(w^{2}\right)}$ and a germ of a local holomorphic $\operatorname{map} \varphi: \mathbb{C}^{n} \times \overline{\mathbb{C}^{d}} \rightarrow \overline{\mathbb{C}^{n-d}}$ such that the germ

$$
\mathbb{C}^{n} \times \overline{\mathbb{C}^{d}} \rightarrow \mathcal{M}, \quad\left(z, \bar{w}^{1}\right) \mapsto\left(z, \bar{w}^{1}, \varphi\left(z, \bar{w}^{1}\right)\right),
$$

is biholomorphic. Then the germ

$$
\begin{aligned}
\Phi: \mathbb{C}^{n} \times \overline{\mathbb{C}^{d}} \times \mathbb{C}^{d} \times \cdots \times \overline{\mathbb{C}^{d}} \rightarrow \mathcal{M}^{s} \\
\quad\left(z_{1}, \bar{w}_{1}^{1}, \ldots, z_{l}^{1}, \bar{w}_{l}^{1}\right) \mapsto \\
\left.\left(z_{1}, \bar{w}_{1}^{1}, \varphi\left(z_{1}, \bar{w}_{1}^{1}\right)\right), z_{2}^{1}, \bar{\varphi}\left(\bar{w}_{1}^{1}, \varphi\left(z_{1}, \bar{w}_{1}^{1}\right), z_{2}^{1}\right), \ldots, \bar{w}_{l}^{1}, \varphi\left(\ldots, \bar{w}_{l}^{1}\right)\right),
\end{aligned}
$$

is also biholomorphic, where $s=2 l-1$. Furthermore, the subset $\mathcal{M}_{x}^{s}$ is equal to $\Phi\left(\left\{z_{1}=x_{1}\right\}\right)$. This proves the lemma. 
Lemma 4.2. Under the assumptions of Proposition 3.1, for every integers $s \geq$ $1, l \geq 0, m:=s r+l$, there exist open neighborhoods

$$
U\left(j_{x}^{m} f_{0}\right) \subset J^{m}\left(\mathbb{C}^{n}, \mathbb{C}^{n^{\prime}}\right), \quad U(x, \bar{x}, \ldots, \bar{x}) \subset \mathcal{M}^{s}
$$

and for every $\nu=\left(z, \bar{w}_{1}, \ldots, z_{l}, \bar{w}\right) \in \mathcal{M}^{s}$, a family of holomorphic maps

$$
F_{\nu}^{l}: U_{z}\left(j_{x}^{m} f_{0}\right) \rightarrow J_{\bar{w}}^{l}\left(\overline{\mathbb{C}^{n}}, \overline{\mathbb{C}^{n^{\prime}}}\right)
$$

such that for all admissible germs $f$ at $x$ with $j_{x}^{m} f \in U\left(j_{x}^{m} f_{0}\right)$,

$$
j_{\bar{w}}^{l} \bar{f}=F_{\nu}^{l}\left(j_{z}^{m} f\right)
$$

where $z, w$ and all $z_{j}, w_{j}$ are sufficiently close to $x$. Moreover, $F_{\nu}$ depends holomorphically on $\nu \in \mathcal{M}^{s}$.

Proof. The required maps $F_{\nu}^{l}, \nu \in \mathcal{M}^{s}$, are obtained as iterates of (38):

$$
F_{\nu}(j):=\overline{F_{\left(w_{l}, \bar{z}_{l+1}\right)}^{l}} \circ F_{\left(z_{l}, \bar{w}_{l}\right)}^{r+l} \circ \cdots \circ F_{\left(z_{1}, \bar{w}_{1}\right)}^{(s-1) r+l}(j) .
$$

Here we iterate step by step the map in (38) and its conjugate. Notice that the conjugate is taken exactly for $z_{j}$ 's and $w_{j}$ 's conjugated to the coordinates of $\nu$. Hence $F_{\nu}$ depends holomorphically on $\nu \in \mathcal{M}^{s}$. The required formula (68) for admissible germs is obtained by iterating (38).

4.2. Segre sets. The right-hand side in (68) depends on $\nu$ which contains several coordinates $z_{j}, w_{j}$ other than $z$ and $w$. To avoid this ambiguity we project out the auxiliary coordinates. The corresponding projection of $\mathcal{M}^{s}$ is a family of the so-called Segre sets introduced in [1]. The family of Segre sets

$$
Q^{2 l+1} \subset \mathbb{C}^{n} \times \overline{\mathbb{C}^{n}}, Q^{2 l} \subset \mathbb{C}^{n} \times \mathbb{C}^{n}
$$

is defined as the projection on the product of the fist space $\mathbb{C}^{n}$ and the last one (which is either $\overline{\mathbb{C}^{n}}$ or $\mathbb{C}^{n}$ ) of $\mathcal{M}^{s}$ for $s=2 l+1$ or $s=2 l$ respectively.

Notice that, on the contrary to the family of Segre varieties $Q_{w}=Q_{w}^{1}$, the family of Segre sets $Q_{w}^{s}$ for $s>1$ is not analytic in general and depends on the neighborhood, where $\mathcal{M}$ is taken. A smaller neighborhood of $(x, \bar{x}) \in \mathcal{M}$ induces a family of smaller Segre sets in each neighborhood of $(x, \bar{x})$.

Let

$$
Q_{w}^{s}:=\left\{z \in \mathbb{C}^{n}:(z, \bar{w}) \in Q^{s}\right\}
$$

be the $s$-th Segre set associated with $w \in \mathbb{C}^{n}$. We make use of the following basic properties of the Segre sets (see [1], §2.2, in particular Corollary 2.2.2):

Theorem 4.1 (Baouendi, Ebenfelt, Rothschild). Let $M \subset \mathbb{C}^{n}$ be a realanalytic $C R$ submanifold. Then the dimension of $Q_{z}^{s}$ increases strictly until it stabilizes. If $M$ is minimal at $x \in M$, then $Q_{x}^{s}$ contains an open subset of $\mathbb{C}^{n}$ for s sufficiently large. 
Since

$$
\operatorname{dim} Q_{w}^{1}=\operatorname{dim} Q_{w}=\operatorname{dim}_{\mathrm{CR}} M,
$$

we can take $s=d+1$, where $d=\operatorname{codim}_{\mathrm{CR}} M$. By Theorem 4.1, the generic rank of the projection

$$
\mathcal{M}_{x}^{d+1} \rightarrow \overline{\mathbb{C}^{n}}
$$

is $n$. Unfortunately this is true only generically whereas the rank of (73) at $(x, \bar{x}, \ldots, \bar{x})$ is usually not maximal. Hence we cannot lift $Q_{x}^{s}$ to $M$ in a neighborhood of $x$ but we shall do this in an open set of $\overline{\mathbb{C}^{n}}$ with $\bar{x}$ on the boundary.

4.3. Liftings of the Segre sets to the iterated complexification. Our next goal will be to obtain a formula similar to (68) where the family of holomorphic maps is parametrized by $w \in Q_{z}^{s}$ instead of $\nu \in \mathcal{M}^{s}$. For this we choose carefully open subsets of the Segre sets $Q_{z}^{s}$ (see (71)) and lift them simultaneously to $\mathcal{M}_{z}^{s}$. The pullbacks of (67) under these liftings will yield the required formulae.

We start with some elementary lemmata which we prove here for convenience of the reader.

Lemma 4.3. Let $X, Y$ be complex manifolds, $x_{0} \in X, \operatorname{dim} X=\operatorname{dim} Y=n \geq 1$, $Y^{\prime} \subset Y$ a submanifold and $f: X \rightarrow Y$ a holomorphic map with $y_{0}:=f\left(x_{0}\right) \in Y^{\prime}$. Suppose that $f$ is of the maximal rank $n$ on a dense subset of $f^{-1}\left(Y^{\prime}\right)$. Then there exists an open subset $N \subset Y$ and a holomorphic lifting $\psi: N \rightarrow X$ (i.e. $f \circ \psi=\mathrm{id}_{N}$ ) such that the following is satisfied:

(1) $N \cap Y^{\prime}$ is connected;

(2) $y_{0}$ is in the closure of $N \cap Y^{\prime}$;

(3) $\psi$ extends continuously to $y_{0}$ with $\psi\left(y_{0}\right)=x_{0}$.

Proof. Without loss of generality, $X$ is an open subset in $\mathbb{C}^{n}$. Set

$$
D:=\left\{x \in X: \operatorname{rank}_{x} f<n\right\} .
$$

Since $D \subset X$ is an analytic subset and $D \cap f^{-1}\left(Y^{\prime}\right)$ is nowhere dense, there exists a local holomorphic curve $C \subset f^{-1}\left(Y^{\prime}\right)$ such that $C \cap C \subset\left\{y_{0}\right\} \subset C$. Then the restriction of $f$ to $C$ is a ramified covering given by $z \mapsto z^{k}$ with respect to some local coordinates. Therefore there exists a real curve

$$
\gamma:[0,1] \rightarrow C, \quad \gamma(0)=x_{0}, \quad f \text { is injective on } \gamma[0,1] .
$$

Let $t_{0} \in[0,1)$ be minimal with the property that there exist $N$ and $\psi$ satisfying (1.) and (3.) in this lemma and such that

$$
f(\gamma(t)) \subset N \text { for } t \in\left(t_{0}, 1\right] .
$$

By the construction of $\gamma, t_{0}$ exists. It is sufficient to show that $t_{0}=0$. 
Suppose that $t_{0}>0$. Since $\operatorname{rank}_{\gamma\left(t_{0}\right)} f=n, f$ is locally biholomorphic at $\gamma\left(t_{0}\right)$. Therefore the lifting $\psi$ can be uniquely extended to a neighborhood $U$ of $f\left(\gamma\left(t_{0}\right)\right)$. Set $N_{1}:=N \cup U$ and further

$$
N_{2}:=\left\{y \in N_{1}: d(\psi(y), \gamma[0,1])<d\left(\psi(y), f^{-1}\left(y_{0}\right)\right)\right\},
$$

where $d$ is the euclidean distance with respect to the ambient coordinates of $\mathbb{C}^{n}$. Then $N_{2}$ and $\psi$ satisfy (3.). By passing to a smaller neighborhood of $f\left(\gamma\left[t_{0}, 1\right]\right)$ we also obtain (1.). Hence $t_{0}$ is not minimal and we have a contradiction.

The following lemma is an elementary fact from linear algebra.

Lemma 4.4. Let $A_{1}: V \rightarrow W_{1}, A_{2}: V \rightarrow W_{2}$ be linear maps between vector spaces such that

(1) $A_{1}$ is surjective;

(2) $A_{2} \mid \operatorname{Ker} A_{1}$ is surjective.

Then the direct sum $A_{1} \oplus A_{2}: V \rightarrow W_{1} \oplus W_{2}$ is also surjective.

In the following lemma let $Y_{1}, Y_{2}, Y_{3}$ be complex manifolds, $X \subset Z:=Y_{1} \times$ $Y_{2} \times Y_{3}$ a submanifold and $a=\left(a_{1}, a_{2}, a_{3}\right) \in X$. Denote by $\pi_{k}$ the natural projection from $X$ to $Y_{k}, k=1,2,3$. Further we use the notation

$$
X_{a}^{1}:=\left\{z \in X: z_{1}=a_{1}\right\}, \quad X_{a}^{2}:=\left\{z \in X: z_{2}=a_{2}\right\}
$$

and similarly $N_{a}^{1}, N_{a}^{2}$ for $N \subset Y_{1} \times Y_{2}$.

Lemma 4.5. Suppose that $X_{a}^{1}$ is a submanifold and for all $z$ from a dense subset of $X_{a}^{1} \operatorname{rank}_{z} \pi_{1}=\operatorname{dim} Y_{1}$ and $\operatorname{rank}_{z}\left(\pi_{2} \mid X_{a}^{1}\right)=\operatorname{dim} Y_{2}$. Then there exist an open subset $N \subset Y_{1} \times Y_{2}$ and a holomorphic lifting $\psi: N \rightarrow X\left(\right.$ i.e. $\left.\left(\pi_{1} \times \pi_{2}\right) \circ \psi=\operatorname{id}_{N}\right)$ such that the following is satisfied:

(1) $N_{a}^{1}$ is connected;

(2) $\left(a_{1}, a_{2}\right)$ is in the closure of $N_{a}^{1}$;

(3) $\psi$ extends continuously to $\left(a_{1}, a_{2}\right)$ with $\psi\left(a_{1}, a_{2}\right)=a$.

Proof. Fix some local coordinates in $Z$ near $a$ (independently of the product structure) such that $X$ and $X_{a}^{1}$ equal to the unit balls in corresponding linear subspaces. By the assumptions, there exists $z \in X_{a}^{1}, z_{2} \neq a_{2}$, such that

$$
\operatorname{rank}_{z} \pi_{1}=\operatorname{dim} Y_{1}, \quad \operatorname{rank}_{z}\left(\pi_{2} \mid X_{a}^{1}\right)=\operatorname{dim} Y_{2} .
$$

By Lemma 4.4, $\operatorname{rank}_{z}\left(\pi_{1} \times \pi_{2}\right)=\operatorname{dim} Y_{1}+\operatorname{dim} Y_{2}$ and therefore the fibers

$$
\left(\pi_{1} \times \pi_{2}\right)^{-1}\left(w_{1}, w_{2}\right)
$$

through $w$ are smooth and of constant dimension for $w \in X$ close to $z$.

We claim that $z$ with this property can be chosen such that $a$ does not lie in the tangent subspace at $z$ to the fiber $\left(\pi_{1} \times \pi_{2}\right)^{-1}\left(z_{1}, z_{2}\right)$ (the tangent subspace is understood as an affine subspace of the ambient coordinate space). Otherwise $a$ would lie in every fiber which contradicts the assumptions. 
Therefore there exists a linear subspace $L \subset Y_{1} \times Y_{2} \times Y_{3}$ through $a$ and $z$ which is transverse and of complementary dimension to the fiber $\left(\pi_{1} \times \pi_{2}\right)^{-1}\left(z_{1}, z_{2}\right)$. Then $\operatorname{rank}_{z}\left(\pi_{1} \times \pi_{2}\right)=\operatorname{dim}\left(Y_{1} \times Y_{2}\right)$.

In addition, we can choose $L$ transverse to $X$ and $X_{a}^{1}$ at $a$. Since $X_{a}^{1}$ is a ball, $L \cap X_{a}^{1}$ is connected. Hence $\operatorname{rank}_{w}\left(\pi_{1} \times \pi_{2}\right)=\operatorname{dim}\left(Y_{1} \times Y_{2}\right)$ for all $w$ from a dense subset of $L \cap X_{a}^{1}$.

Then $f:=\left(\pi_{1} \times \pi_{2}\right) \mid(L \cap X)$ together with $Y^{\prime}:=\left\{a_{1}\right\} \times Y_{2} \subset Y:=Y_{1} \times Y_{2}$ satisfies the assumptions of Lemma 4.3. The lemma follows now directly from Lemma 4.3.

4.4. Applications of liftings. As the next step we apply the above lemmata to our situation. Again we assume that $s:=d+1$ is odd. Set $b:=(x, \bar{x}) \in \mathbb{C}^{n} \times \overline{\mathbb{C}^{n}}$.

Lemma 4.6. Under the assumptions of Theorem 1.2 for all integers $s \geq 1$, $l \geq 0, m:=s r+l$, there exist an open subset $N \subset \mathbb{C}^{n} \times \overline{\mathbb{C}^{n}}$, an open neighborhood $U\left(j_{x}^{m} f_{0}\right) \subset J^{m}\left(\mathbb{C}^{n}, \mathbb{C}^{n^{\prime}}\right)$ and for $(x, \bar{w}) \in N$, holomorphic maps

$$
\varphi^{l}: U_{x}\left(j_{x}^{m} f_{0}\right) \times N_{b}^{1} \rightarrow J^{l}\left(\overline{\mathbb{C}^{n}}, \overline{\mathbb{C}^{n^{\prime}}}\right), \quad \Phi_{\bar{w}}^{l}: \overline{U_{w}\left(j_{x}^{m} f_{0}\right)} \times N_{(x, \bar{w})}^{2} \rightarrow \mathbb{C}^{n^{\prime}}
$$

such that the following is satisfied:

(1) $N_{b}^{1}$ is connected;

(2) $b$ is in the closure of $N_{b}^{1}$;

(3) for all admissible germs $f$ at $x$ with $j_{x}^{m} f \in U\left(j_{x}^{m} f_{0}\right)$,

$$
j_{\bar{w}}^{l} \bar{f}=\varphi^{l}\left(j_{x}^{m} f, \bar{w}\right), \quad j_{z}^{l} f=\Phi_{\bar{w}}^{l}\left(j_{\bar{w}}^{m} \bar{f}, z\right),
$$

for $(z, \bar{w}) \in N$ sufficiently close to $(x, \bar{x})$.

Moreover, the map $\Phi_{\bar{w}}^{l}$ depends holomorphically on $\bar{w}$.

Proof. Put in Lemma 4.5

$$
X:=M^{s} \subset \mathbb{C}^{n} \times \mathbb{C}^{N} \times \overline{\mathbb{C}^{n}}, \quad Y_{1}:=\mathbb{C}^{n}, \quad Y_{2}:=\overline{\mathbb{C}^{n}}, \quad Y_{3}:=\mathbb{C}^{N}
$$

and fix the point $a:=(x, \ldots, \bar{x}) \in X$. By Lemma $4.1, X$ and $X_{a}^{1}$ are manifolds and the first condition $\operatorname{rank}_{z} \pi_{1}=\operatorname{dim} Y_{1}$ in Lemma 4.5 is satisfied. The second condition $\operatorname{rank}_{z}\left(\pi_{2} \mid X_{x}^{1}\right)=\operatorname{dim} Y_{2}$ is satisfied by the choice of $s$ which we made by Theorem 4.1. Let

$$
N \subset \mathbb{C}^{n} \times \overline{\mathbb{C}^{n}}, \quad \psi: N \rightarrow M^{s}
$$

be given by Lemma 4.5. Statements (1.) and (2.) in this lemma follow directly from (1.) and (2.) in Lemma 4.5 respectively. It remains to satisfy (3.).

Let

$$
F_{\nu}^{l}: U_{z}\left(j_{x}^{m} f_{0}\right) \rightarrow J_{\bar{w}}^{l}\left(\overline{\mathbb{C}^{n}}, \overline{\mathbb{C}^{n^{\prime}}}\right)
$$

be as in Lemma 4.2. Passing if necessary to a smaller subset $N$ define

$$
\varphi^{l}(j, \bar{w}):=F_{\psi(x, \bar{w})}^{l}(j)
$$


for all $j \in U_{x}\left(j_{x}^{m} f_{0}\right),(x, \bar{w}) \in N_{x}^{1}$. By (3.) in Lemma 4.5, $\varphi^{l}$ satisfies the required properties. Similarly define

$$
\Phi_{\bar{w}}^{l}(\bar{j}, z):=\bar{F}_{\bar{\psi}(\bar{w}, z)}^{l}(\bar{j}) .
$$

4.5. The end of the proof. The last step is based on combining the equations in (82). Let $f_{0}, f$ be as in Theorem 1.2 and set

$$
m:=s r=(d+1) r, \quad k:=2 m .
$$

We further use the notation of Lemma 4.6. The set $N_{b}^{1}$ can be seen in the canonical way as an open subset of $\mathbb{C}^{n}$. In general it may not contain $\bar{x}$. Combining the equations in (82) we obtain

$$
f(z)=\Phi_{\bar{w}}^{m}\left(\varphi^{m}\left(j_{x}^{k} f, \bar{w}\right), z\right),
$$

where $(z, \bar{w}) \in N$ is sufficiently close to $(x, \bar{x})$.

Recall that we denoted by $S$ the set of all admissible germs at $x$ (see Definition 1.5). For the proof of Theorem 1.2 we have to show conditions (1.),(2.),(3.) in Definition 1.2. Suppose that $j_{x}^{k} f=j_{x}^{k} f_{0}$. Then (1.) is followed by (89).

Denote by $\Phi$ the map in the right-hand side of (89):

$$
\Phi: U_{x}\left(j_{x}^{k} f_{0}\right) \times N \rightarrow \mathbb{C}^{n^{\prime}}, \quad \Phi(j, z, \bar{w}):=\Phi_{\bar{w}}^{m}\left(\varphi^{m}(j, \bar{w}), z\right) .
$$

Then (89) can be rewritten as

$$
f(z)=\Phi\left(j_{x}^{k} f, z, \bar{w}\right) .
$$

The left-hand side in (91) is defined for $z$ close to $x$. The right-hand side is defined for $j_{x}^{k} f \in U\left(j_{x}^{k} f_{0}\right)$ and $(z, \bar{w}) \in N$. To show (2.) and (3.) in Definition 1.2 we fix some $\bar{w}_{0} \in N_{b}^{1}$.

Lemma 4.7. For every admissible $f$ with $j_{x}^{k} f \in U\left(j_{x}^{k} f_{0}\right)$, there exists a neighborhood $U(x) \subset \mathbb{C}^{n}$ such that (91) is valid for all $z \in U(x)$ and for $w=w_{0}$.

Proof. Since $(91)$ is valid for all $(z, \bar{w}) \in N$ close to $(x, \bar{x})$, we can choose it in the form $\left(x, \bar{w}_{1}\right)$ because of $\left(2\right.$.) in Lemma 4.6. Since $N_{b}^{1}$ is connected, there exists a real curve $\gamma \subset N_{b}^{1}$ which connects $\bar{w}_{1}$ with $\bar{w}_{0}$. Since $\gamma$ is compact, there exists a connected product neighborhood

$$
\{x\} \times \gamma \quad \subset \quad U(x) \times U(\gamma) \quad \subset \quad N .
$$

Without loss of generality, $f(z)$ is defined for all $z \in U(x)$.

Hence both sides of (91) are defined for $z \in U(x), \bar{w} \in U(\gamma)$. By the choice of $\bar{w}_{1}$, they are equal in a neighborhood of $\left(x, \bar{w}_{1}\right)$. The conclusion of the lemma follows from the identity principle.

By Lemma 4.7, for $f$ fixed and $z$ close to $x$

$$
f(z)=\Phi\left(j_{x}^{k} f, z, \bar{w}_{0}\right), \quad z \in U(x) .
$$


The right-hand side is defined for

$$
j_{x}^{k} f \in U\left(j_{x}^{k} f_{0}\right), \quad z \in N_{\left(x, \bar{w}_{0}\right)}^{2}
$$

independently of $f$. Hence all such admissible germs $f$ extend to the open set $N_{\left(x, \bar{w}_{0}\right)}^{2}$ which proves (2.).

Finally define

$$
F(j, z):=\Phi\left(j, z, \bar{w}_{0}\right), \quad F: U_{x}\left(j_{x}^{k} f_{0}\right) \times N_{\left(x, \bar{w}_{0}\right)}^{2} \rightarrow \mathbb{C}^{n^{\prime}} .
$$

By the choice of $w_{0}$ one has $x \in N_{\left(x, \bar{w}_{0}\right)}^{2}$. Then (3.) is implied by (93). This finishes the proof of Theorem 1.2.

\section{Acknowledgements}

I am very grateful to K. Diederich for calling my attention to the jet method and to M. S. Baouendi, P. Ebenfelt and L. P. Rothschild for their valuable corrections and remarks.

\section{References}

1. M. S. Baouendi, P. Ebenfelt, and L. P. Rothschild, Algebraicity of holomorphic mappings between real algebraic sets in $\mathbb{C}^{n}$, Acta Math. 177 (1996), 225-273.

2. — CR automorphisms of real analytic manifolds in complex space, Comm. Anal. Geom., (to appear), 1997.

3. - Parametrization of local biholomorphisms of real analytic hypersurfaces, Asian J. Math. 1 (1997), 1-16.

4. M. S. Baouendi, H. Jacobowitz, and F. Treves. On the analyticity of CR mappings, Ann. of Math. (2) 122 (1985), 365-400.

5. V. K. Beloshapka, A uniqueness theorem for automorphisms of a nondegenerate surface in the complex space, Math. Notes, 47 (1990), 239-242.

6. H. Cartan, Sur les groupes de transformations analytiques, Act. Sc. et Int. Hermann, Paris, 1935.

7. S. S. Chern and J. K. Moser, Real hypersurfaces in complex manifolds, Acta Math. 133 (1974), 219-271.

8. K. Diederich and J. E. Fornæss, Proper holomorphic mappings between real analytic pseudoconvex domains, Math. Ann. 282 (1988), 681-700.

9. K. Diederich and S. Webster, A reflection principle for degenerate real hypersurfaces, Duke Math. J. 47 (1980), 835-843.

10. H. Lewy, On the boundary behaviour of holomorphic mappings, Accad. Naz. Lincei 35 (1977), 1-8.

11. R. Narasimhan, Several complex variables, Chicago Lectures in Mathematics, Univ. of Chicago Press, 1971.

12. S. I. Pinchuk, On the analytic continuation of holomorphic mappings, Math. USSR Sbornik 27 (1975), 345-392.

13. B. Segre, Intorno al problem de Poincaré della representazione pseudoconform, Rend. Accad. Lincei 13 (1931), 676-683.

14. N. Tanaka, On the pseudo-conformal geometry of hypersurfaces of the space of $n$ complex variables, J. Math. Soc. Japan 14 (1962), 397-429.

15. A. E. Tumanov, Extending CR functions on manifolds of finite type to a wedge, Math. Sbornik 136 (1988), 129-140. 
16. S. Webster, On the mapping problem for algebraic real hypersurfaces, Invent. Math. 43 (1977), 53-68.

17. 71 (1978), 26-28.

18. D. Zaitsev, On the automorphism groups of algebraic bounded domains, Math. Ann. 302 (1995), 105-129.

Mathematisches Institut, Universität TüBIngen, 72076 TÜBIngen, GERMANY

E-mail address: dmitri.zaitsev@uni-tuebingen.de 